\title{
Oculomotor Paresis with Cyclic Spasms: Description of Four Cases and Hypothesis of the Mechanism
}

\author{
Haitian Nan and Yun Jing* \\ Department of Neurology, Capital Medical University, China
}

*Corresponding author: Yun Jing, Department of Neurology, Beijing Tongren Hospital, Capital Medical University, China

\begin{abstract}
Oculomotor paresis with cyclic spasms (OPCS) is a rare and usually congenital disorder. It typically presents at birth or within the first 2 years of life. Acquired OPCS resulting from aneurysm compression on the nerve and after irradiation therapy has been reported. OPCS is characterized by unilateral third nerve paresis with periodic spasms causing eyelid elevation, miosis, and contraction of 1 or more of the extraocular muscles innervated by the third nerve. Here we report four patients with this rare ocular condition and propose possible mechanisms and differential diagnoses for their presentation. In case of patient \#4 the symptoms secondary to gamma knife radiosurgery is confusingly similar to that of Ocular neuromyotonia (ONM). The association of COPS and gamma knife radiosurgery is hitherto unreported in the literature. It seems likely that COPS may share some similarity on peripheral mechanisms of ONM.
\end{abstract}

\section{Introduction}

Oculomotor Paresis with Cyclic Spasms (OPCS) is a rare disorder characterized by periodic paralysis alternating with spasms of complete or partial ocular muscles innervated by the third nerve. During a paretic phase, the patient with OPCS has unilateral ptosis, mydriasis, and limitation of adduction. In a spastic phase, the patient has ipsilateral eyelid lifted, miosis, limitation of abduction. These spasms last 10 to 30 seconds and then turn to the paretic phase. The majority of patients with OPCS present in infancy. A patient developed constant cycles of the third nerve paresis and spasm after radiation therapy. Since the original review of 2 case of OPCS by Zhang FH in Beijing Tongren Hospital in 1984 we present here four more cases of this disorder in the same hospital and propose possible mechanisms for their presentation.

\section{Case Report}

\section{Case 1}

A 23-year-old man was referred with a right repetitive ptosis and exotropia since birth and developed evident symptoms at age 5. He visited Neurologist in Beijing Tongren hospital in January, 2010. At presentation, his visual acuity was $0.4=20 / 50$ OU, near acuity vision was Jr1 OU. Anterior segment examination and fundus were normal. Right ptosis with half cornea covered, exotropia $30^{\circ}$, limitation of adduction, turning up and down were noticed. Examination revealed cyclic paralysis and spasm of right oculomotor nerve. During a 20-25 second of paretic phase (SDC00184 0:2$0: 25 \mathrm{sec}$ ), the patient had right ptosis, mydriasis, pupillary light response disappeared and exotropia $30^{\circ}$ with variable limitation of looking in, up and down. After 2 second (0:25-0:26sec) of upper eyelid shiver, spastic phase succeeded with 25 second(0:26$50 \mathrm{sec}$ ) of ipsilateral lid retraction, myosis, pupillary light response appearing and the globe turning to the midline. After final 1 second (51sec) of upper eyelid fibrillation, the cycles entered next turn. Right palpebral fissure was $3 \mathrm{~mm}$ and the pupil diameter was $5 \mathrm{~mm}$ in the paretic phase, then the palpebral fissure was $11 \mathrm{~mm}$ and the pupil diameter was $2 \mathrm{~mm}$ in the spastic phase. On primary position the contralateral fissure measured $9 \mathrm{~mm}$ and contralateral pupil diameter measured $5 \mathrm{~mm}$. The patient accentuated that tension and sustained reading at near favored the extent and duration of the spastic symptoms, as a supplement. Physical evaluation and head MRI gave normal results. Based on the patient's history, imaging, and clinical examination, OPCS was diagnosed (Table 1). 
Table 1: summary of clinical characteristics of four cases. $\left(^{*}\right)$ The patient had right amblyopia with exotropia.

\begin{tabular}{|c|c|c|c|c|}
\hline Characteristics & Case1 & Case 2 & Case 3* & Case 4 \\
\hline Sex & Male & Female & Female & Female \\
\hline Age, year & 23 & 22 & 15 & 63 \\
\hline Onset age, year & Birth & $<1$ & 9 & 63 \\
\hline Side & Right & Right & Left & Right \\
\hline Visual acuity & $\begin{array}{l}20 / 50(\mathrm{OU}) \\
\mathrm{J} 1(\mathrm{OU})\end{array}$ & $\begin{array}{c}\text { 20/100(OU) } \\
\text { J7(OD); J1(OS) }\end{array}$ & $\begin{array}{c}\text { 20/200(OD);20/40(OS) } \\
\mathrm{J}<7(\mathrm{OD}) ; \mathrm{J} 2(\mathrm{OS})\end{array}$ & $\mathrm{J} 6(\mathrm{OD}) ; \mathrm{J} 6(\mathrm{OS})$ \\
\hline \multicolumn{5}{|c|}{ Paretic Phase } \\
\hline Ptosis, mm & 3 & 3 & 4 & 5 \\
\hline Mydriasis, mm & 5 & 6 & 5 & 3 \\
\hline Exotropia, $^{\circ}$ & 30 & 10 & 30 & 0 \\
\hline Duration & $20-25$ seconds & $20-25$ seconds & $15-20$ seconds & 30minutes \\
\hline \multicolumn{5}{|c|}{ Spastic Phase } \\
\hline Upper eyelid retraction, $\mathrm{mm}$ & 11 & 8 & 10 & 7 \\
\hline Miosis, mm & 2 & 2 & $2-3$ & 2 \\
\hline Duration, seconds & $25-30$ & $15-25$ & $15-20$ & $20-30$ \\
\hline \multicolumn{5}{|c|}{ Contralateral Eye } \\
\hline Eyelid fissure, mm & 9 & 8 & 7 & 7 \\
\hline Pupil, mm & 5 & 5 & 5 & 2 \\
\hline
\end{tabular}

\section{Case 2}

A 22-year-old girl presented to Neuro-Ophthalmology Center, Beijing Tongreng Hospital in August 2009, with a chief complaint of right ptosis and exotropia. She developed a right ptosis and exotropia during the first year of her life. Past medical history was notable for surgery for right ptosis at 1-year-old and squint surgery at 15-year-old. At presentation, her distance visual acuity was 20/100 in both eyes (OU), near visual acuity was Jr7 OD and Jr1 OS. Anterior segment examination and left fundus were normal, right fundoscopy showed optic disc border clear, temporal color pale. On this occasion, right cyclic oculomotor palsy and spasms were seen. In the paralytic phase, she had right ptosis with a palpebral fissure of $3 \mathrm{~mm}, 6 \mathrm{~mm}$-diameter of mydriasis, pupillary light response weakened and a mild exotropia. Paralytic phase lasted for approximately 20 to 25 seconds. (SDC10441 0:12sec$0: 37 \mathrm{sec}$; 0:53-1:15. In the following spastic phase, right eyelid quivering for about 2 seconds (SDC10441 0:38sec-0:39sec,1:15-16, then lifting to $8 \mathrm{~mm}, 2 \mathrm{~mm}$-diameter of myosis and mild esotropia were witnessed. The spastic phase lasted for approximately 1525 seconds (SDC10441 39sec-53sec,1:16-1:31) then entered next turn. On primary position, left palpebral fissure measured $8 \mathrm{~mm}$ and left pupil measured 5mm-diameter. Cycles were not affected by voluntary gaze. Systematic examination was normal. Brain MRI demonstrated no abnormality. On the basis of the patient's history, imaging, and clinical examination, OPCS concurrent with right amblyopia was diagnosed.

\section{Case 3}

A 15-year-old girl complained of left blepharoptosis was upon presentation to our clinic 6 years after the symptoms observed and 2 and half months after the symptoms got worse. At the age of 9 because of an emotional blow (her grandfather died), she had left blepharoptosis, ophthalmoplegia, and eyelid tremor, without a fatigue phenomenon. The symptoms were exacerbated sometimes when she was in angry or anxious since then. Two and a half months ago, the patient felt left blepharoptosis, ophthalmoplegia and eyelid quiver aggravated after undergoing a sleepless night of palpitation, sweating, and limb trembling when her grandmother died. At presentation in November 2008, her distance vision acuity was $0.1=20 / 200$ OD and $0.5=20 / 40$ OS, near acuity vision was Jr 7 OD and Jr2 OS. Her left palpebral fissure and left pupil changed cyclically. In the paretic phase of a cycle, she had left ptosis, left blepharophimosis with a palpebral fissure of $4 \mathrm{~mm}$ and a half pupil covered. In addition, she had exotropia of $30^{\circ}$, internal rotation from the inner canthus of $4 \mathrm{~mm}$, limitation up and down, pupil expanded to diameter of $5 \mathrm{~mm}$, and weak pupillary reflexes to light, which phase lasted for approximately 15 to 20 seconds. In the spastic phase, the ipsilateral palpebral fissure lifted to $10 \mathrm{~mm}$, eyeball back to the midline, pupil down to $2-3 \mathrm{~mm}$ with sensitive pupillary light response were witnessed. The spastic phase lasted approximately 15 to 20 seconds. On primary position the palpebral fissure measured $7 \mathrm{~mm}$ on the right eye, right pupil diameter measured $5 \mathrm{~mm}$, and the right eye was exotropia $40^{\circ}$ and fully activated in all directions. The systematic examination was normal. Laboratory examinations showed FT3 10.01pmol/L (reference value $2.6 \sim 6.8 \mathrm{pmol} / \mathrm{L}$ ), FT4 $34.38 \mathrm{pmol} / \mathrm{L}$ (reference value $10 \sim 22 \mathrm{pmol} / \mathrm{L}$ ), TSH $0.01 \mathrm{mIU} / \mathrm{mL}$ (reference value $0.27 \sim 4.2 \mathrm{mIU} /$ $\mathrm{mL}$ ). Neostigmine test and repetitive nerve stimulation revealed no abnormalities. Head MRI showed cerebellar tonsillar herniation, in line with Arnoild-Chiair deformation type I. We gave her diagnoses 
of OPCS, Arnoild-Chiair deformation type I, hyperthyroidism, right amblyopia with exotropia.

\section{Case 4}

A 63-year-old female complained of cyclic esotropia was upon presentation to our clinic 3 months after the symptoms observed. 3 months ago, she found her right eyeball moved inside gradually and then developed diplopia. This symptom occurred cyclically every 30 minutes and lasted for 20 seconds until her right eyeball moved back to midline within 10 seconds. At presentation on October 2014, her near acuity vision was Jr6 OD and Jr6 OS. During the spastic phase, the patient had right esotropia, myosis of $2 \mathrm{~mm}$, sensitive pupillary light response, and reduced scardamyxis. The ipsilateral palpebral fissure was $7 \mathrm{~mm}$. The spastic phase lasted for 20 seconds. Then the globe moved toward midline within 10 seconds and then entered paretic phase. In the paretic phase, the patient had neither limitation of eye movements nor diplopia. Physical examination of eye movements showed nothing abnormal. Whereas, her right palpebral fissure reduced to $5 \mathrm{~mm}$, with ipsilateral ptosis, $3 \mathrm{~mm}$ diameter of mydriasis, pupillary light response weakened. In both spastic phase and paretic phase, other neurological systematic examination was normal. On primary position the palpebral fissure measured $7 \mathrm{~mm}$ on the left eye, left pupil diameter measured $2 \mathrm{~mm}$. 6 years ago the patient had gamma knife radiation therapy for suprasellar meningoma. This time after admission we scheduled cavernous sinus MRI for the patient. It revealed an abnormality around the right cavernous segment of internal carotid artery. An aneurysm with thrombosis from the right cavernous segment of internal carotid artery was considered. On the basis of the patient's symptom, imaging, and clinical examination, acquired oculomotor nerve paresis with cyclic spasm was diagnosed. We suggested the patient to complete Digital Subtraction Angiography for further vascular assessment. The patient refused and her symptoms remained the same when she discharged automatically.

\section{Discussion}

The ocular changes described in the four patients here are in line with the definition of OPCS which is characterized by alternating rhythmic paralysis and spasm of all or part of ocular muscles innervated by oculomotor nerve.1 2 Four patients displayed unilateral ptosis, mydriasis, light response weakened, and the first three patients displayed exotropia in the paretic phase. In the following spasticity, they presented ipsilateral eyelid elevation, which was comparable to the contralateral lid in patient \#1\& \#4 and over-elevated in \#2 \& \#3, miosis, and the globe wandered to middle position or esotropia. Paretic phases last approximately 15-25 seconds, spastic phases last approximately 15-30 seconds, and entire cycles last approximately 30-55 seconds. Most cases described have been present at birth or in infancy. Distinctively, the paretic phase of patient \#4 lasts approximately 30 minutes. In addition, her symptom was present at the age of 63 , that is, 6 years after radiation therapy for suprasellar meningoma.
Patient \#3 had the disease onset at age 9 because of extreme grief and was concurrent with Arnoild-Chiair deformation type I, right amblyopia and right exotropia. Since this syndrome is speculated to be in association with birth trauma or early damage, [1] we assume this patient might have been overlooked for her ocular pathology of both eyes in her early life and just been connected the onset of the disease with a memorable event. In this condition, we may draw the conclusion that her unaffected eye was amblyopia with exotropia. Emotional stimulations and sympathetic hyperactivity made patient \#3 feel the cyclic symptoms aggravating time and again, and mental stress made patient \#2 feel the spastic symptoms worse. It is reported that the rate and amplitude of the cyclic movements could be influenced by physiologic conditions, such as sleep, arousal, physical or emotional stress, systemic drugs, and could be accelerated when the patients were stirred up by mental or emotional stress [1].

The diagnose Oculomotor nerve paresis with cyclic spasms should be differentiated from some diseases, such as ocular neuromyotonia [2-7], brain trauma [8], tumor, inflammation [8] or aneurysm [5], ocular myasthenia, demyelinating diseases, Graves' disease, migraine, or intracranial hypertension. Spontaneous, brief, recurrent unilateral oculomotor nerve palsies lasting from 30 seconds to 4 hours over the period of 2 to 4 years have been reported in 3 otherwise normal individuals. These 3 patients were onset without any known provocation and any sequela of systemic or neurological disorder. Different from cyclic oculomotor nerve palsy and spasms [9], these 3 patients were normal between episodes, and did not present cyclic paresis and spasms. Consecutive cyclic exotropia has been described after surgical correction of cyclic esotropia which was assumed as a complication of brachytherapy for choroidal melanoma in the same person [10]. The condition differs from oculomotor nerve paresis with cyclic spasms, in that the patient did not show paresis during esotropia cycles or exotropia cycles. Intermittent involuntary monocular eyelid elevation with complete ptosis had been described in patients undergone surgery of ipsilateral sphenoid wing meningioma. The patients developed ophthalmoplegia and decreased corneal sensation and could elevate the lid by either tickling the eyelashes or rubbing the eyelids. Unlike oculomotor paresis with cyclic spasms, the lid opening was provoked by a manual stimulus, rather than cyclic in nature. It is speculated that the ability to elevate a paralyzed lid without volitional effort is associated with aberrant regeneration activated by sensory stimuli [11]. Some patient developed oculomotor "misdirection synkinesia" from recovery from basal third nerve lesion by trauma or aneurysms. When the patient attempted to moving the globe up, down, or in, the eyelid lifting, the globe adduction, and pupillary constriction occurred in the eye innervated by impaired third nerve. The innervating pattern of "misdirection synkinesia" is due to indiscriminate growing of the injured nerve fibers during the recovery and reaching the muscles not supplied formerly. Unlike oculomotor Nerve Palsies and cyclic 
spasms, the patient with "misdirection synkinesia" showed the lid lifted, the pupil constricted immediately at the start of the action, instead of after a long latent period of looking up, down and in [1]. Another disorder suggested by our patients is ocular neuromyotonia. Ocular neuromyotonia (ONM) is characterized by paroxysmal spontaneous contraction of one or more ocular motor muscles innervated by either the oculomotor, trochlear, or abducens nerves. Half of patients with Ocular neuromyotonia suffered from radiation to the parasellar and sella regions and presented the symptoms in 2 months to 18 years after therapy [4]. Compressive lesions on ocular motor nerves, such as internal carotid artery aneurysm, Graves orbitopathy have also been implicated, some were accepted as idiopathic [7]. Damage to ocular motor nerves due to local radiation and neurovascular compression are the main cause of the disease. The definite pathophysiology of ONM is unknown. Shults and associates [5] hypothesized that unstable membranes of injured ocular motor axons may generate spontaneous impulses, which produce involuntary sustained and inappropriate ocular muscle contraction, thus leading to periods of spasm between which ocular motility and alignment are normal [12]. Sporadic case reports followed supported the theory that ocular neuromyotonia could result from ephaptic transmission, whereby damaged cranial nerve axons cause aberrant neural signaling, most likely as the result of injured or demyelinated cell membranes [7]. Gordon T. Plant further theorized the physiology of neuromyotonia as a disorder of cell membrane potassium channels, such that the axon can no longer maintain membrane stability. During sustained muscle activity, extracellular potassium accumulates and provokes neuromyotonic discharges [13]. In 2014, Deepak Menon, etc. reported two cases of ONM caused by brainstem demyelination. This further emphasized the pathophysiology of ONM as secondary to segmental demyelination [14], which conduce to cell membranes of nucleus neurons or axons unstable. Thus, carbamazepine has often been approved effectiveness in treating ocular neuromyotonia as a membrane stabilizer of hyperexcitable axons.

Although ocular neuromyotonia partially has some features in common with cyclic oculomotor paresis and spasms, there are differences between the two entities. Unlike cyclic oculomotor palsy, ocular neuromyotonia causes paroxysmal spasm of the muscles supplied by oculomotor nerve for the duration of the episodes, but no paresis persists between these episodes, unless the patient has a preexisting acquired or congenital strabismus. The episodes of spasm can be triggered by sustained gaze in a certain direction in two thirds of patients of Ocular neuromyotonia, whereas the episodes of cyclic oculomotor paresis and spasms occur spontaneously [2,4]. Reportedly, COPS could be secondary to the compression on the nerve caused by aneurysm in dorsum sella [15] and irradiation of the skull base [2]. It is noticeable that patient \#4 could be misdiagnosed as neuromyotonia (ONM) for her typical symptoms in spastic phase. She had right esotropia during the spastic phase and showed no sign of exotropia during the paralytic phase. However, by carefully physical examination, we noticed that she had myosis of $2 \mathrm{~mm}$, sensitive pupillary light response, and reduced scardamyxis in the spastic phase. By comparison, her right palpebral fissure reduced to $5 \mathrm{~mm}$, pupillary light response weakened during the paralytic phase. $3 \mathrm{~mm}$ diameter of mydriasis was also found during her paralytic phase. These partially oculomotor paralytic symptoms occur subsequently after spastic phase suggest that the patient cannot be diagnosed as ocular neuromyotonia. Since the symptoms occur cyclically every 30 minutes, we believe the condition was more consistent with an acquired form of cyclic oculomotor nerve paresis and spasms, characterized by continuous cycles of paresis and spasm of the oculomotor nerve that were neither induced nor altered by eccentric gaze.

In 2004, Neil R. Miller and Andrew G. Lee reported two cases of adult-onset left oculomotor nerve paresis with cyclic spasm. Both patients had a history of radiation therapy, and the symptoms cycled at regular 2-minute to 4-minute intervals between a left oculomotor nerve paresis and spasm of the oculomotor nerve. Both the patients had an oculomotor nerve paresis with ptosis, mydriasis, reduced accommodation, and ophthalmoparesis induced by exotropia. About every 2-4 minutes, the ptotic eyelid elevates, the globe begins to adduct, the pupil constricts, and accommodation increases. In coordinate with the findings of Miller NR, patient \#4 had radiation therapy for suprasellar meningoma 6 years before the onset of this disease. The only distinction is that she had no exotropia or ophthalmoparesis during the paralytic phase. In common with Miller NR's cases, there was no history of preexisting oculomotor nerve paresis or diplopia in patient \#4, there was no evidence of aberrant regeneration of the oculomotor nerve during the paretic phases of the cycle, the paresis was characterized by both ptosis and papillary changes, and both the spastic and paretic phases of the cycle were, as noted previously, continuous and of predictable length.

Most cases of oculomotor nerve paresis with cyclic spasm occurred in children or in adults with a long history of some type of ocular motor dysfunction in childhood. The pathogenesis of acquired COPS is unclear but probably similar to that of ONM. Loewenfeld and Thompson [1] believed that both congenital and acquired COPS were caused by damage to the intracranial portion of the oculomotor nerve, after which retrograde degeneration of oculomotor neurons occurred, damaging both the oculomotor nerve and its nucleus, the nerve by the primary process, and the nucleus by secondary retrograde degeneration. This, in turn, led to the survival of two populations of neurons, those that were injured and those that were hyperactive. The damage of the oculomotor nerve caused by radiotherapy of patient \#4 could lead to the cyclic symptom, despite that the cyclic symptom might as well be caused by the compression of aneurysm from the right cavernous segment of internal carotid artery. However, our case and the cases reported by MILLER have one thing in common: COPS occured 
under the same condition, that is, after radiation of the skull base. Gadoth, Avi etc. also reported a 34-year-old woman with intermittent episodes of vertical and horizontal diplopia associated with intermittent incomplete closure of the left eyelid in 2013 [16]. The symptoms were onset 5 years after brain radiotherapy for cerebellar medulloblastoma. The episodes consisted of partial cyclic oculomotor spasms and paresis of the left eye. They lasted for 1-2 minutes, recurred every few hours spontaneously or provoked by focusing on an object. Brain Magnetic Resonance Imaging (MRI) with contrast showed no evidence of recurrent tumor. Treatment with carbamazepine $400 \mathrm{mg}$ daily completely relieved the eye movement disorder. In similarity, although the possibility of the compression of aneurysm cannot be completely ruled out, we believe that radiotherapy play a more important role in the cause of patient \#4. It seems likely that cyclic oculomotor nerve paresis with spasms may share some similarity on peripheral mechanisms of ONM, by which damaged oculomotor nerve cause aberrant regeneration and consequent ephaptic transmission [17]. Together with the patients reported by Miller NR and Gadoth Avi, the clinical findings in patien\#4 combining COPS with neuromyotonic characteristics further support this theory.

Over half of the cases of ocular neuromyotonia reported have been associated with conventional external irradiation of the sellar/ parasellar region. To date, only few cases of ocular neuromyotonia caused by Gamma Knife Radiosurgery (GKS) has been reported, whereas no case of cyclic oculomotor nerve paresis with spasms caused by GKS has been reported. Much JW reported a patient of ocular neuromyotonia following treatment of GKS in 2009 [18]. W.C. Candy Sze described a series of three patients in whom a clinical diagnosis of ONM was made 3-6 months following delivery of GKS to the sellar/parasellar region [19]. They concluded although gamma knife radiosurgery differs from conventional external beam pituitary irradiation, it also has the possibility to play an etiological role in ONM. Patient \#4 was treated with Gamma Knife Radiosurgery (GKS) for suprasellar meningioma 6 years before her symptoms developed. Our case suggests that GKS could also lead to cyclic oculomotor nerve paresis with spasms, which may share some similarity on peripheral mechanisms of ONM. Hitherto this is the first case of COPS reported after gamma knife radiosurgery. The diagnosis of COPS should be considered in patients with history of GKS who complain of intermittent blurring or diplopia in clinical practice.

The patient \#2 had typical symptoms of cyclic oculomotor palsy and spasms except that sustained reading at near could exacerbate the spastic performance. Although it is unusual, it is not a rare phenomenon. 31 of 54 patients with cyclic oculomotor palsy have been described in whom adduction or accommodation convergence action aggravated spastic extent and duration; in the meantime, abduction efforts enhanced the paretic phase [1]. Arnoild-Chiair deformation type I in patient \#3 might be a supporting factor for a central mechanism involved. It was reported that the third nerve palsy was attributed to deformation of the brainstem [14,20]. The lesion may destruct the sympathetic inhibitory path from hypothalamus to the dilator muscle of pupil or degenerate the oculomotor neuron. In turn, it led to hyperactive or injured neurons. Besides, the acceleration of rate and amplitude of the spastic phase elicited by mental stress in patient \#2, as well as the influence of sympathetic hyperactivity following emotional strike upon the bouts of the disease in Patient \#3 would support the assumption of the central mechanism. The cyclic spasms that were unmasked with sympathetic overaction suggest disinhibition of central control. We agree with the opinion proposed by Loewenfeld IE, Thompson HS, that together with the third nerve, the oculomotor nucleus and its central connections may be involved in Oculomotor nerve paresis with cyclic spasms [1]. There is no special treatment for Oculomotor nerve paresis with cyclic spasms. Squint surgery, such as recession of lateral rectus and medial rectus, levator shorting or lid suspension procedures, can correct the exotropia and ptotic eyelid, whereas the procedures have no effect on the cyclic phases, especially cyclical changes in the pupil [3]. Besides, surgeries for ptosis may give rise to risks of corneal exposure [21]. Currently, there is no proven treatment for the cyclic spasms.

\section{References}

1. Loewenfeld IE, Thompson HS (1975) Oculomotor paresis with cyclic spasms. A critical review of the literature and a new case. Survey of Ophthalmology 20(2): 81-124.

2. Miller NR, Lee AG (2004) Adult-onset acquired oculomotor nerve paresis with cyclic spasms: Relationship to ocular neuromyotonia. American Journal of Ophthalmology 137(1): 70-76.

3. Zhang Fang hua (1984) Cyclic oculomotor spasm and relaxation phenomenon report of 2 cases. Chin Med J 97(11): 858-860.

4. Alexandre de Saint Sardos, Angela Vincent, Maryam Aroichane, Luis H, Ospina de Saint Sardos et al. (2008) Ocular neuromyotonia in a 15-yearold girl after radiation therapy. Journal of AAPOS Journal of American Association for Pediatric Ophthalmology and Strabismus 12(6): 616617.

5. Hee Young Park, Jeong-Min Hwang b, Ji Soo Kim (2008) Abducens neuromyotonia due to internal carotid artery aneurysm. Journal of the Neurological Sciences 270(1-2): 205-208.

6. Chung SM, Lee AG, Holds JB, Roper-Hall G, Cruz OA (1997) Ocular neuromyotonia in Graves dysthyroid orbitopathy. Arch Ophthalmol 115(3): 365-370.

7. Betigul Yürüten, Süleyman IIhan (2003) Ocular neuromyotonia: A case report Clin Neurol Neurosurg 105(2): 140-142.

8. Menon V, Arya AV, Prakash P. Pupillary (1989) automatism Indian J Ophthalmol 37(1): 30.

9. Michael S Lee, Robert A Egan, William T Shults, Simmons Lessell (2005) Idiopathic Repetitive Oculomotor Nerve Palsies in Otherwise Normal Patients. Ophthalmology 112(12): 2225-2226.

10. Sunir J Garg, Steven M Archer (2007) Consecutive cyclic exotropia after surgery for adult-onset cyclic esotropia. Journal of AAPOS 11(4): 412413.

11. Robert A Egan, Judith EA (2006) Intermittent reversal of complete ptosis associated with sphenoid wing meningiomas. Can J Ophthalmol 41(4): 497-499. 
12. Shults WT, Hoyt WF, Behrens M (1986) Ocular neuromyotonia. A clinical description of six patients. Arch Ophthalmol 104(7): 1028-1034.

13. Gordon T Plant (2006) Putting Ocular Neuromyotonia in Context. Journal of Neuro-Ophthalmology 26(4): 241-243.

14. Menon D, Sreedharan SE, Gupta M, Nair (2014) A novel association of ocular neuromyotonia with brainstem demyelination: Two case reports. Mult Scler 20(10): 1409-1412.

15. Bateman DE, Saunders M Cyclic (1983) Oculomotor palsy: Description of a case and hypothesis of the mechanism. J Neurol Neurosurg Psychiatry 46(5): 451-453.

16. Gadoth A, Kipervasser S, Korczyn AD, Neufeld MY, Kesler A (2013) Acquired Oculomotor Nerve Paresis with Cyclic Spasms in a Young Woman, a Rare Subtype of Neuromyotonia. J Neuro-Ophthalmol 33(3): 247-248.

17. Choi KD, Hwang JM, ParkSH, Kim JS (2006) Primary aberrantregeneration and neuromyotonia of the third cranial nerve. J Neuroophthalmol 26(4): 248-250.
18. Plant GT (2006) Putting ocular neuromyotonia in context. J Neuroophthalmol 26(4): 241-243.

19. Much JW, Weber ED, Newman SA (2009) Ocular neuromyotonia after gamma knife stereotactic radiation therapy. Journal of NeuroOphthalmology 29(2): 136-139.

20. Sze WC, McQuillan J, Plowman PN, MacDougall N, Blackburn P, et al. (2015) Stereotactic radiosurgery XX: Ocular neuromyotonia in association with gamma knife radiosurgery. Endocrinol Diabetes Metab Case Rep. 140106.

21. Winterkorn JM, Bruno M (2001) Relative pupil-sparing oculomotor nerve palsy as the presenting sign of posterior fossa meningioma. J Neuroophthalmol 21(3): 207-209.

22. Yazici B, Ünal M, Köksal M, Özdek SC (2000) Oculomotor palsy with cyclic spasms: A case report. Orbit 19(2): 129-133.

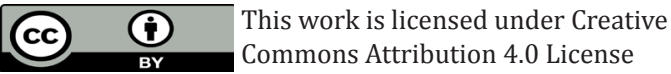

To Submit Your Article Click Here:

Submit Article

DOI: $10.32474 / 0 J N B D .2019 .02 .000150$

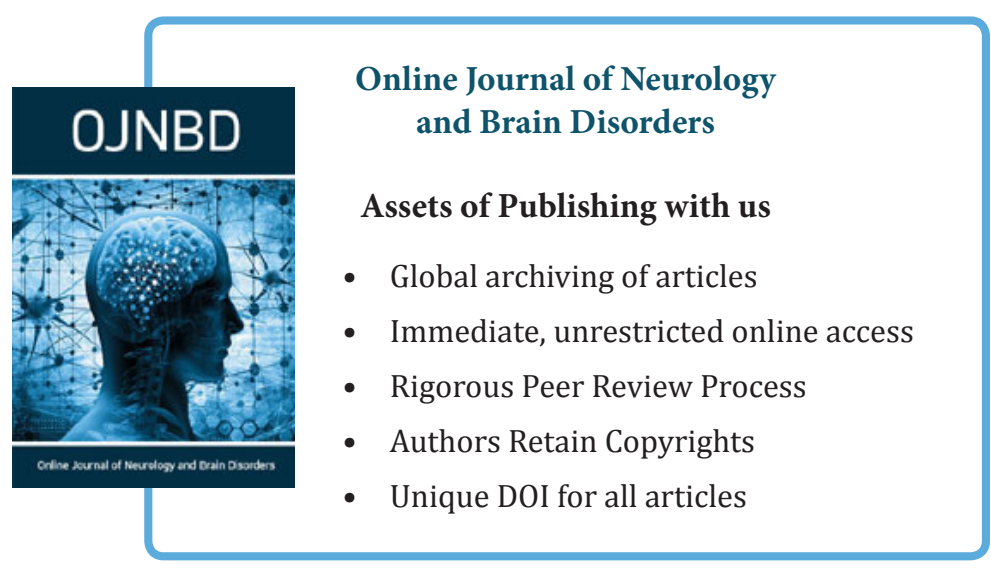

